Review

\title{
Application of the Chick Embryo Chorioallantoic Membrane in Neurosurgery Disease
}

\author{
Yong-Jie Yuan*, Kan $\mathrm{Xu}^{*}$, Wei Wu, Qi Luo ${ }^{\bowtie}$, and Jin-Lu Yu ${ }^{\bowtie}$ \\ Department of Neurosurgery, Jilin University First Hospital, Changchun 130021, China. \\ * These authors contributed equally to this work.
}

$\triangle$ Corresponding authors: Jin-Lu Yu and Qi Luo, Department of Neurosurgery, Jilin University First Hospital, Changchun 130021, China. Tel: +86-431-88782264 Email: jlyu@jlu.edu.cn.

(c) Ivyspring International Publisher. This is an open-access article distributed under the terms of the Creative Commons License (http://creativecommons.org/ licenses/by-nc-nd/3.0/). Reproduction is permitted for personal, noncommercial use, provided that the article is in whole, unmodified, and properly cited.

Received: 2014.08.30; Accepted: 2014.10.13; Published: 2014.10.27

\begin{abstract}
The chick embryo chorioallantoic membrane (CAM) is a highly vascularized extraembryonic membrane. Because of its ease of accessibility, extensive vascularization and immunodeficient environment, the CAM has been broadly used in the oncology, biology, pharmacy, and tissue regeneration research. The present review summarizes the application of the CAM in neurosurgery disease research. We focused on the use of the CAM as an assay for the research of glioma, vascular anomalies, Moyamoya Disease, and the blood-brain barrier.
\end{abstract}

Key words: chorioallantoic membrane, glioma, vascular disease, blood brain barrier.

\section{Introduction}

The chick embryo chorioallantoic membrane (CAM) is an extraembryonic membrane. Its main function is to exchange gases and nutrients, which is supported by a dense capillary network [1-3]. Due to its extensive vascularization and its ease of use, the CAM is a widely utilized research tool. Because of its extensive vascularization and easy accessibility, morphofunctional characteristics of angiogenesis in vivo as well as the efficacy and mechanisms of action of proand antiangiogenic molecules have been studied using the CAM [2-4]. Due to the lack of a fully developed immune system in the chick embryo [3,5], the CAM is suitable for tumor engraftment to study various aspects of the angiogenic and metastatic potential of human malignancies ${ }^{[3,4]}$, such as glioma $[6-8]$, colorectal cancer [9, 10], leukemia [11], ovarian cancer [4], prostate cancer [12, 13], and osteosarcoma[14]. In addition, the CAM has been used to test the biocompatibility of organic and inorganic materials [15-17], the ability of bacterial strains to invade epithelial barriers [18], vascular anomalies and Moyamoya Disease [19-24], the organogenetic capacity of xenograft or homograft $[25$,
26], the mechanisms of wound healing [27, 28], and the differentiation of the blood-brain barrier [29,30]. The aim of the present review is to summarize the application of the CAM in the field of neurosurgery research. We focused on the use of the CAM as a model for the research of glioma, vascular anomalies, Moyamoya Disease, and the blood-brain barrier.

\section{General structure and function of the CAM}

Chick embryo development lasts 21 days. At 3.5 days of incubation, the allantois of the chick embryo appears as an evagination from the ventral wall of the endodermal hindgut. At 4.5 days of incubation, the allantois pushes out of the body of the embryo into the extraembryonic coelom. Its proximal portion lies parallel and caudal to the yolk sac. The narrow proximal portion is the allantoic stalk and is wrapped around the umbilical artery and umbilical vein. The distal portion is full of allantoic fluid, and gradually separates from the embryo and enlarges. This is the allantoic vesicle, which rapidly enlarges from day 
four to day ten. Fluid accumulation distends the allantois so that its terminal portion resembles a balloon $[2,3,31]$. An extensive morphometric investigation has shown rapid extension of the CAM surface area from $6 \mathrm{~cm}^{2}$ at day six to $65 \mathrm{~cm}^{2}$ at day 14 [32]. In this process, the mesodermal layer of the allantois fuses with the adjacent mesodermal layer of the chorion to form the CAM. The CAM adheres to the acellular inner shell membrane that almost surrounds the entire embryo and other egg contents [2, 3,31,33]. Histologically, the CAM consists of three layers, the chorionic epithelium, which is the somatic mesoderm attached to the shell membrane, the allantoic epithelium which is the splanchnic mesoderm facing the allantoic cavity, and the intermediate mesodermal layer, which is between the chorionic and allantoic epithelium and enriched in blood vessels and stromal components (Fig. 1) $[2,3,31,33]$.

The general vascular system of the CAM consists of the capillary plexus located between the chorionic epithelium and allantoic epithelium, and the larger umbilical artery and vein within the allantoic vesicle (Fig. 2) [34]. The umbilical artery emerges from the embryonic abdominal wall, branches into two primary chorioallantoic arteries, and then into six or seven generations. The earlier fifth and sixth generations of blood vessels are parallel to the CAM surface and the later generations change direction abruptly, passing almost vertically in the two-dimensional capillary plexus. The capillary plexus is drained by a single chorioallantoic vein to the embryo [35, 36].

The CAM blood vessels appear at day four as a network of immature, irregularly spaced tubules with large luminal diameters, scattered in the mesoderm between two layers of epithelium. The CAM vessels grow rapidly until day eight. Then gradually differentiate into the capillary plexus, arterioles, and venules [31,35]. These immature blood vessels lack a complete basal lamina and smooth muscle cells (SMC), so there is little ultrastructural distinction between capillaries, arterioles, and venules. At nine to thirteen days, arterial endothelial junctions become more extensive, and presumptive SMC and connective tissue become apparent [35,37]. Capillaries show no changes in the periendothelial space and veins accumulate SMC and connective tissue to a lesser extent than arteries. Arteries have developed a distinct muscular layer and a more complete basement membrane by day 14 to day $18[35,37,38]$.

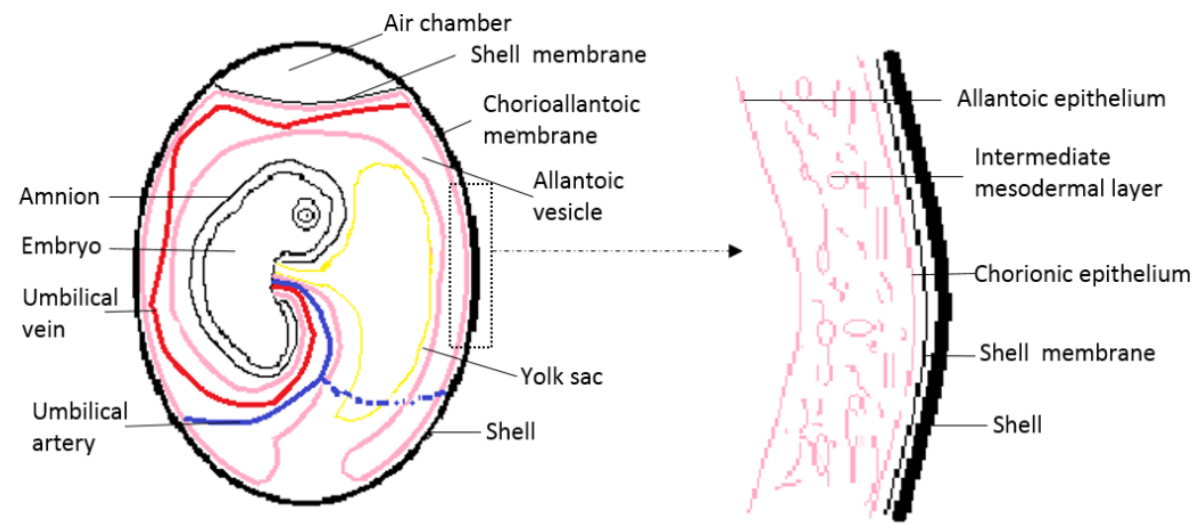

Figure I. Schematic drawing of the general structure of a 12 day old chick embryo in the egg and the three-tissue layers of the chick chorioallantoic membrane (CAM).
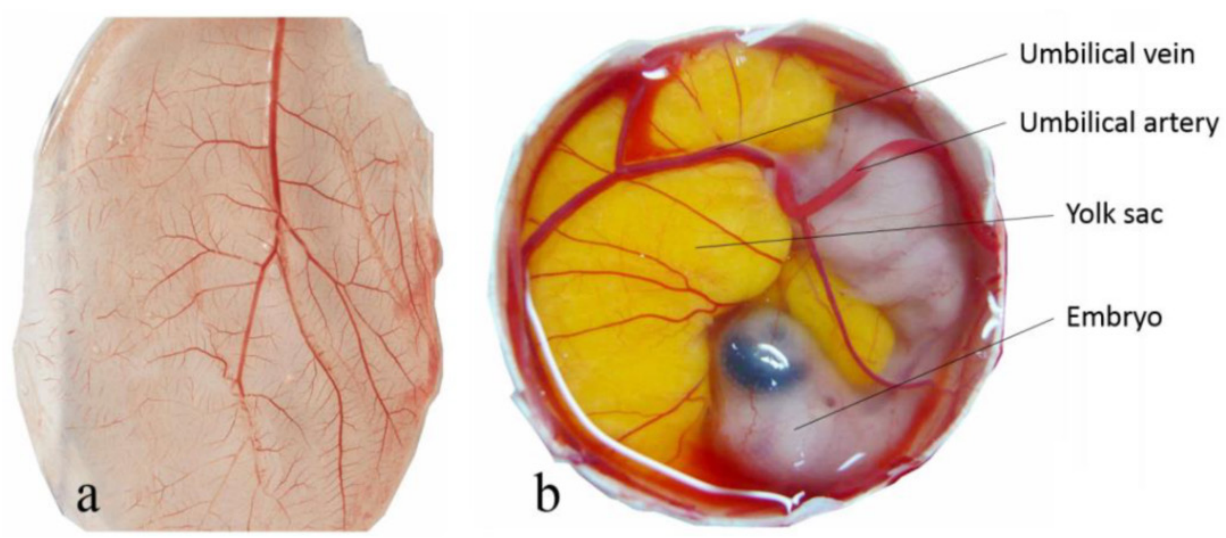

Figure 2. The general vascular system of the chorioallantoic membrane (CAM). Capillary plexus of CAM of a 10 day old chick embryo (a) and the umbilical atery and umbilical vein free-floating within the allantoic vesicle (b). 
The CAM has a very simple vascularized structure, but serves multiple functions during embryo development. The capillary plexus and the CAM confer a respiratory function [2,39-41]. In addition to the respiratory interchange of oxygen and carbon dioxide, it is involved in the calcium transport from the eggshell [42-46], acid-base homeostasis in the embryo [31,47, 48], and ion and water reabsorption from the allantoic fluid [49,50]. The allantois also serves as reservoir for the waste products excreted by the embryo $[2,34]$.

\section{Application of the CAM in glioma re- search}

The CAM is especially well suited for xenograft experiments due to its immune-deficient environment and dense capillary network [51]. Hurst, et. al. [52] first demonstrated that a human tumor of the central nervous system (meningioma) could be successfully transplanted to the CAM. Since then, Vogel, et. al. [53] directly transplanted numerous intracranial tumors, including cerebellar astrocytoma, glioblastoma multiforme, meningioma, and craniopharyngioma to the CAM. Seventy-one percent of the eggs initially survived the procedure, and $41 \%$ of the tumors were viable after one week. Microscopically, the first generation of the inoculated tumor resembled the parent tumors. Upon subsequent transplantation, specimens were necrotic and did not survive past the second or third generation ${ }^{[53]}$.

Tereseviciute, et. al. [54] and Balciuniene, et. al. [51] evaluated the histology and survival of human glioblastoma tumors transplanted to the chicken CAM. The studies showed that glioblastoma cells not only survived in the CAM, but also appeared histologically similar to the original glioblastoma. Surprisingly, the xenograft's immune cells (e.g. macrophages) also survived. Tereseviciute observed invasion of avian vessels in the xenograft [54]. However, Balciuniene observed that the transplanted glioblastoma remained an insulated unit that survived from nourishment of the chorioallantoic membrane by diffusion. They also observed minimal invasion of the tumor cells into chicken tissue [51].

Strojnik, et. al. [55] compared the CAM glioma and rat glioma models. The full ranges of cytological features of human glioblastoma were observed in both models. In the rat glioma model, the xenograft tumors were sharply demarcated from the surrounding brain tissue. In the CAM glioma model, smaller tumor nodules were observed growing within the connective tissue and vessel walls. Therefore, Strojnik, et.al. proposed that the CAM glioma model may be more suitable for tumor invasiveness studies than the rat glioma model [55]. Furthermore, the CAM glioma model is a popular choice to investigate glioma angi- ogenesis, growth, invasiveness, and the screening of antitumor drugs because it is less expensive, has a shorter experimental duration, has a simple protocol, and has minor ethical concerns compared to the rat glioma model.

\section{The CAM as an effective model to study glioma angiogenesis and antiangi- ogenesis}

Angiogenesis is essential in establishing a network of blood vessels needed to supply oxygen and nutrients required for promoting the survival and invasiveness of glioma cells. Glioma cells release specific growth factors to activate endothelial cell growth of the host tissue in order to become highly vascularized [56]. Increased vessel density around the xenograft has been repeatedly confirmed in the CAM glioma model [1,57-59]. Therefore, the CAM glioma model has been widely used to assess the effects of drugs or cell factors on angiogenesis. Table 1 summarizes the studies described in this section.

Table I. The chick CAM as a model to study angiogenesis and antiangiogenesis of glioma.

\begin{tabular}{|c|c|c|}
\hline Author & Intervention & Intervention effect \\
\hline Hagedorn [59] & RTKi & Antiangiogenesis \\
\hline Auf G [65] & Silencing IRE1 & Antiangiogenesis \\
\hline Nico B [58] & Anti-Epo & Antiangiogenesis \\
\hline Pen A[60] & IGFBP7 & Antiangiogenesis \\
\hline Jiang L[63] & Bmi-1-overexpressing & Angiogenesis \\
\hline Martinho O[57] & RTKi & Antiangiogenesis \\
\hline Miranda-Goncalves $\mathrm{V}^{[8]}$ & $\mathrm{CHC}$ & Antiangiogenesis \\
\hline $\mathrm{He} Y Q^{[56]}$ & DMPP & Antiangiogenesis \\
\hline
\end{tabular}

Abbreviations: RTKi: receptor tyrosine kinase inhibitors; IRE1: inositol-requiring enzyme 1a; Epo: erythropoietin; IGFBP7: insulin-like growth factor binding protein

7; Bmi-1: B-cell-specific Moloney murine leukemia virus integration site 1; CHC:

Alpha-cyano-4-hidroxycinnamate; DMPP: dimethyl phenyl piperazine iodide.

Dimethyl phenyl piperazine iodide (DMPP) is antiangiogenic. In a dose-dependent manner, U87 glioma cell xenografts treated with DMPP had less growth and angiogenesis compared with the untreated cells [56]. However, DMPP did not inhibit U87 cell proliferation in vitro, even at high concentration. This implies that the tumor regression observed in the xenografts was due to DMPP blocking angiogenesis [56].

Gliomas exhibit high glycolytic rates, and monocarboxylate transporters (MCTs) play a major role in the maintenance of the glycolytic metabolism through the proton-linked transmembrane transport of lactate. In the CAM glioma model, Alpha-cyano-4hidroxycinnamate (CHC, MCT inhibitor) induced a decrease in the tumor perimeter and the number of blood vessels compared with the untreated group. 
However, treatment of the untransplanted CAM with $\mathrm{CHC}$ does not affect the angiogenesis of the CAM, indicating that $\mathrm{CHC}$ impaired tumor glycolytic metabolism ${ }^{[8]}$.

Martinho, et. al. utilized the CAM glioblastoma model to study the effect of receptor tyrosine kinase inhibitors (RTKi) on angiogenesis and tumor proliferation in vivo. They observed that the RTKi reduced the number of blood vessels recruited to tumors and inhibited tumor growth. Combined with additional in vitro experiments, they identified new receptor tyrosine kinase (RTK) targets of RTKi. The CAM glioblastoma model could be used to identify potential predictive biomarkers to anti-RTK therapies and provide a basis for specific targeted therapies in the future [57].

Insulin-like growth factor binding protein 7 (IGFBP7) is a tumor suppressor gene, which has been proposed to participate in senescence, apoptosis and late phase angiogenesis of tumor cells [60]. After treatment of the CAM U87 glioma model with IGFBP7, the sizes of formed vessels around the tumor were reduced by $40 \%$ compared with untreated tumors. Immunohistochemical examination confirmed that the presence of IGFBP7 promoted the recruitment and differentiation of vessel pericytes, which stabilized the tumor blood vessels and suppressed tumor growth [60].

Erythropoietin (Epo) is a cytokine with pleiotropic effects, which exhibit a wide variety of biological functions in non-hematopoietic tissues, such as angiogenesis [61]. It has been observed that the expression of Epo is greatly increased in high-grade gliomas compared to normal brain, but the biological function was unclear [62]. To determine whether Epo was stimulating angiogenesis in the gliomas, Nico, et. al. treated the CAM glioma model with an anti-Epo antibody. They observed that angiogenesis was inhibited, suggesting that Epo may play a role in glioma angiogenesis [58].

The CAM glioma model was also used to evaluate gene regulation on tumor angiogenesis. B-cell-specific Moloney murine leukemia virus integration site 1 (Bmi-1), an onco-protein, has been implicated in the progression of gliomas. Jiang, et. al. observed that Bmi-1-overexpressing glioma cells induced more second- and third-order vessels in the CAM model compared to control cells. This was due to the activation of the NF-kB signaling pathway by Bmi-1. NF-kB induces expression of vascular endothelial growth factor C (VEGF-C), which plays a pivotal role in tumor vascularization [63, 64].

Auf, et. al. inoculated inositol-requiring enzyme 1a (IRE1) dominant-negative U87 cells on the CAM. They observed the size of the tumor was significantly smaller and tumor angiogenesis was decreased compared with control cells. However, the IRE1 dominant-negative U87 cells also exhibited a highly invasive aspect arising from the tumor masses and spreading along blood and lymphatic vessels inside the CAM. The overall structure of the chicken membrane appeared disorganized compared to the control cells [65]. This may suggest that some antiangiogenic therapy may have limited benefits in the treatment of glioma.

\section{The CAM as a model to study glioma invasion}

The CAM glioma model has been utilized to study the invasion potential of glioma (Table 2). The research has focused on the invasion of the chorionic epithelium and the blood vessels by the tumor cells. The transplanted glioma cells or specimens invaded the chorionic epithelium and grew into the mesenchymal connective tissue. Invasion into neighboring vessels and metastasis to distant organs has also been observed [1, 6, 54,59, 66]. Hagedorn, et. al. established a glioma progression model using the CAM. Glioma cell invasion into the CAM was clearly visible by immunostained sections. The CAM blood vessels served as guiding structures for migration of the glioma cells. Moreover, several cancer-progression genes (e.g. CYR61, LUM) were up regulated after $48 \mathrm{~h}$ post-implantation ${ }^{[59]}$.

Table 2. The chick CAM as a model to study glioma invasion.

\begin{tabular}{lll}
\hline Author & Intervention & Intervention effect \\
\hline Westhoff $[6]$ & NF-kB inhibition & Anti-invasion \\
Auf G ${ }^{[65]}$ & Silencing IRE1 & Anti-invasion \\
Teruszkin ${ }^{[66]}$ & perillyl alcohol & Anti-metastatic
\end{tabular}

Abbreviations: IRE1: inositol-requiring enzyme 1a

After the establishment of the invasion model, Westhoff, et. al. used the CAM glioma model to demonstrate the importance of transcription factor $\mathrm{NF}-\mathrm{kB}$ in glioma invasion. Inhibition of NF-kB, either by transfection with an IkBa superrepressor (SR) construct or pharmacologically by treatment with Disulfiram, greatly inhibited the invasion of the glioma cells into the CAM [6].

Inhibition of invasion has been studied using both genetic and pharmacological inhibitors. As mentioned previously, silencing of IRE1 in U87 glioma cells exhibited invasion and spreading along blood and lymphatic vessels inside the CAM. The overall structure of the chicken membrane was disorganized. Abnormal hyperplasic vessels were closely associated with the tumor nodules. [65]. Teruszkin, et. al. demon- 
strated the anti-metastatic potential of perillyl alcohol on glioma C6 cells using the CAM model. C6 cells intrinsically metastasize when inoculated in the CAM model. C6 cells treated with concentrations of 0.3 and $0.03 \%$ perillyl alcohol did not invade the lower CAM, whereas the untreated cells did [66].

\section{Other applications of the CAM in gli- oma research}

The CAM glioma model has also been applied to developing new drugs and treatments for individual therapy (Table 3). Warnock, et. al. first imaged tumor glucose metabolism and protein synthesis in the CAM U87 glioblastoma model by positron emission tomography (PET) via catheterization of CAM blood vessels with fludeoxyglucose (18F-FDG). Warnock, et. al. proposed the use of this model to screen tracers and optimize imaging parameters [7].

Table 3. Other applications of CAM in the research of glioma.

\begin{tabular}{ll}
\hline Author & Applications \\
\hline Shoin ${ }^{[6]}$ & $\begin{array}{l}\text { Screening sensitive chemotherapy drugs for the clinical indi- } \\
\text { vidual treatment }\end{array}$ \\
Grodzik[68] & $\begin{array}{l}\text { Testing new anti-glioma drug } \\
\text { Durupt }{ }^{[67]}\end{array}$ \\
$\begin{array}{l}\text { Test anticancer effect of oncolytic adenoviruses } \\
\text { Warnock[7] }\end{array}$ & $\begin{array}{l}\text { Screening of new tracers and optimization of imaging param- } \\
\text { eters }\end{array}$ \\
\hline
\end{tabular}

Durupt, et. al. used the CAM glioma model to test the anticancer effect of oncolytic adenoviruses. Histological and immunohistochemical analyses of tumor sections confirmed large areas of necrosis, adenovirus replication and syncytia formation in the glioma tumors. This suggests that the CAM glioma model is also a promising tool to be used in preclinical evaluation of oncolytic viruses [67]. Carbon nanoparticles have the potential to become a new anti-glioma drug. Grodzik, et. al. showed that carbon nanoparticles inhibited angiogenesis, invasion, fibroblast growth factor-2 (FGF-2) and vascular endothelial growth factor (VEGF) expression [68].

Shoin, et. al. used the CAM glioma model to assess the efficacy of anticancer drugs with clinical effects in 21 patients with previously untreated malignant glioma. They observed an over-all predictive accuracy of $90 \%$ and concluded that the use of the chick CAM model was a good predictive test for tumor sensitivity to chemotherapeutics. [69].

\section{Application of the CAM in vascular dis- ease research}

There is a close relationship between the abnormalities during the embryonic period and the formation of some congenital intracranial vascular anomalies, such as arteriovenous malformation (AVM). The dense capillary network and the characteristic of continuous angiogenesis of the CAM provide a model for studying this vascular anomaly. Jedelska, et. al. successfully cultivated two AVM biopsy specimens on the CAM. Lesions adjacent to the adipose tissues were used as a negative control. Both AVMs induced an angiogenic response that was particularly prominent after four to eight days, and the adipose tissue did not elicit any vascular reaction. Histopathologic examination revealed that the AVM tissue derived from the CAM retained similarity to the tissue of origin and the transplanted AVM tissue was reperfused with the CAM blood. This research showed that AVM can grow reliably on the CAM assay and has the propensity to induce angiogenesis and re-vascularization. The CAM AVM model may be a potential platform for studying the generative mechanism and therapy of AVM [19].

Several authors have demonstrated that injecting mouse aortic endothelial cells transfected with a retroviral expression vector harboring a human FGF2 cDNA (pZipFGF2 MAE cells) into the allantoic sac induced neovascularization and the appearance of hemangioma-like lesions on the CAM. Therefore, the CAM/FGF2-overexpressing pZipFGF2 MAE cell system may be a powerful tool to study the genesis of vascular lesions [19-22, 70].

Moyamoya Disease is a progressive intracranial vascular disorder particularly affecting the circle of Willis and its arteries that results most commonly in transient ischemic attacks ${ }^{[71]}$. An indirect anastomosis operation is one of the effective operations to treat ischemic Moyamoya Disease. The blood supply used may be from different human tissues, such as the anastomotic branches of the scalp arteries, temporal muscle, omentum, galea, or dura. The best tissue is dependent on the ability to produce new vessels. In order to evaluate the adequacy of materials utilized for anastomosis in Moyamoya Disease, Imaizumi, et. al. compared the ability of different human tissues to induce angiogenesis on the CAM. Fresh tissue samples were obtained at the operation and inoculated on the CAM. Neovascularization was evaluated 96 hours later. The scalp artery exhibited the highest activity of angiogenesis, followed by the omentum and galea, while the temporal muscle, dura, and fat tissue showed poor activity [24]. Another study showed that the outer layer of the epidural exhibited significantly higher angiogenic activity than the inner layer of the dura [23]. This research has laid the theoretical foundation for indirect anastomosis in the treatment of ischemic Moyamoya Disease. 


\section{Application of the CAM blood brain bar- rier research}

The CAM has also been applied to study the differentiation of the blood brain barrier (BBB). Janzer, et. al. transplanted purified astrocytes in the CAM and observed that the vessels associated with the grafts are impermeable to Evan's blue, a vascular tracer that does not pass through BBB vessels. However, the vessels associated with the meningeal- or skin-derived fibroblast grafts allowed visible leakage of Evan's blue. This is the first time that the astrocytes induced BBB features in the non-neural origin vessels on the CAM ${ }^{[72] .}$

Lobrinus, et. al. observed that the CAM vessels can express neurothelin, a transmembrane glycoprotein of the immunoglobulin superfamily and a marker of chicken BBB-forming endothelial cells [29, 73], when exposed to astrocyte-conditioned medium but not when exposed to glioma- or endothelial cell-conditioned medium [74]. This suggested that an unknown soluble factor secreted by astrocytes might play an important role in inducing expression of BBB properties in endothelial cells. However, Holash, et. al. observed that mature astrocytes do not induce expression of neurothelin or another molecular marker, the glucose transporter (GT) in the CAM vessels. However, when a fetal chick neural tube was grafted on the CAM, GT was induced in the endothelium [75, 76]. Taken together, astrocyte-induced formation of the $\mathrm{BBB}$ remains controversial.

\section{Conclusion}

In summary, this review represents the application of CAM in the field of neurosurgery disease. The CAM is a valuable alternative to rodent in vivo models for the study of angiogenesis and invasiveness of glioma, gene and protein expression in tumor formation, screening antiglioma drugs, origin and treatment of vascular anomalies, and differentiation of the BBB. Compared with rodent models, the main advantages of the CAM in vivo assays are low cost, simplicity, short experimental duration, easy dynamic observation, minor ethical concerns and a naturally immunodeficient environment. There are some limitations in the application of the CAM, including nonspecific inflammatory reactions and less specific antibodies available. However, the complete sequencing of the chicken genome and the maturity of transgenic chicken technology will be helpful to develop more specific antibodies and give the CAM experimental model more attention.

\section{Acknowledgements}

This work was supported by the National Natural Science Foundation of China (81200888).

\section{Competing Interests}

The authors have declared that no competing interest exists.

\section{References}

1. Cimpean AM, Ribatti D, Raica M. The chick embryo chorioallantoic membrane as a model to study tumor metastasis. Angiogenesis. 2008;11:311-9.

2. Ribatti D. Chick Embryo Chorioallantoic Membrane as a Useful Tool to Study Angiogenesis. Int Rev Cel Mol Bio. 2008;270:181-224.

3. Ribatti D. Chicken chorioallantoic membrane angiogenesis model. Methods in molecular biology. 2012;843:47-57.

4. Lokman NA, Elder AS, Ricciardelli C, Oehler MK. Chick Chorioallantoic Membrane (CAM) Assay as an In Vivo Model to Study the Effect of Newly Identified Molecules on Ovarian Cancer Invasion and Metastasis. International journal of molecular sciences. 2012;13:9959-70.

5. Palmer TD, Lewis J, Zijlstra A. Quantitative analysis of cancer metastasis using an avian embryo model. Journal of visualized experiments : JoVE. 2011.

6. Westhoff MA, Zhou S, Nonnenmacher L, et al. Inhibition of NF-kappaB signaling ablates the invasive phenotype of glioblastoma. Molecular cancer research : MCR. 2013;11:1611-23.

7. Warnock G, Turtoi A, Blomme A, et al. In vivo PET/CT in a human glioblastoma chicken chorioallantoic membrane model: a new tool for oncology and radiotracer development. Journal of nuclear medicine : official publication, Society of Nuclear Medicine. 2013;54:1782-8.

8. Miranda-Goncalves V, Honavar M, Pinheiro C, et al. Monocarboxylate transporters (MCTs) in gliomas: expression and exploitation as therapeutic targets. Neuro-oncology. 2013;15:172-88.

9. Ankri R, Peretz D, Motiei M, Sella-Tavor O, Popovtzer R. New optical method for enhanced detection of colon cancer by capsule endoscopy. Nanoscale. 2013;5:9806-11.

10. Subauste MC, Kupriyanova TA, Conn EM, Ardi VC, Quigley JP, Deryugina EI. Evaluation of metastatic and angiogenic potentials of human colon carcinoma cells in chick embryo model systems. Clinical \& experimental metastasis. 2009;26:1033-47.

11. Taizi M, Deutsch VR, Leitner A, Ohana A, Goldstein RS. A novel and rapid in vivo system for testing therapeutics on human leukemias. Experimental hematology. 2006;34:1698-708

12. Wittig-Blaich SM, Kacprzyk LA, Eismann T, et al. Matrix-dependent regulation of AKT in Hepsin-overexpressing PC3 prostate cancer cells. Neoplasia. 2011;13:579-89

13. Conn EM, Botkjaer KA, Kupriyanova TA, Andreasen PA, Deryugina EI, Quigley JP. Comparative analysis of metastasis variants derived from human prostate carcinoma cells: roles in intravasation of VEGF-mediated angiogenesis and uPA-mediated invasion. The American journal of pathology. 2009;175:1638-52.

14. Balke M, Neumann A, Kersting C, et al. Morphologic characterization of osteosarcoma growth on the chick chorioallantoic membrane. BMC research notes. 2010;3:58.

15. Rickert D, Lendlein A, Peters I, Moses MA, Franke RP. Biocompatibility testing of novel multifunctional polymeric biomaterials for tissue engineering applications in head and neck surgery: an overview. European archives of oto-rhino-laryngology : official journal of the European Federation of Oto-Rhino-Laryngological Societies. 2006;263:215-22.

16. Klueh U, Dorsky DI, Moussy F, Kreutzer DL. Ex ova chick chorioallantoic membrane as a novel model for evaluation of tissue responses to biomaterials and implants. Journal of biomedical materials research Part A. 2003:67:838-43.

17. Spanel-Borowski K. The chick chorioallantoic membrane as test system for biocompatible materials. Research in experimental medicine Zeitschrift fur die gesamte experimentelle Medizin einschliesslich experimenteller Chirurgie. 1989;189:69-75.

18. Adam R, Mussa S, Lindemann D, et al. The avian chorioallantoic membrane in ovo--a useful model for bacterial invasion assays. International journal of medical microbiology : IJMM. 2002;292:267-75.

19. Jedelska J, Strehlow B, Bakowsky U, et al. The Chorioallantoic Membrane Assay Is a Promising Ex Vivo Model System for the Study of Vascular Anomalies. In Vivo. 2013;27:701-05.

20. Liekens S, Neyts J, De Clercq E, Verbeken E, Ribatti D, Presta M. Inhibition of fibroblast growth factor-2-induced vascular tumor formation by the acyclic nucleoside phosphonate cidofovir. Cancer research. 2001;61:5057-64.

21. Dell'Era P, Belleri M, Stabile H, Massardi M, Ribatti D, Presta M. Paracrine and autocrine effects of fibroblast growth factor-4 in endothelial cells. Oncogene. 2001;20:2655-63.

22. Ribatti D, Gualandris A, Belleri M, et al. Alterations of blood vessel development by endothelial cells overexpressing fibroblast growth factor- 2 . The Journal of pathology. 1999;189:590-9. 
23. Kashiwagi S, Kato S, Yasuhara S, Wakuta Y, Yamashita T, Ito H. Use of a split dura for revascularization of ischemic hemispheres in moyamoya disease. Journal of neurosurgery. 1996;85:380-83.

24. Imaizumi T, Hashi K, Nakamura T, Tanabe S. Angiogenic properties of various tissues utilized for synangiosis in moyamoya disease. No shinkei geka Neurological surgery. 1989;17:515-20.

25. Noiman T, Buzhor E, Metsuyanim S, et al. A rapid in vivo assay system for analyzing the organogenetic capacity of human kidney cells. Organogenesis. 2011;7:140-4.

26. Chiba A, Yui C, Hirano S. Liver reconstruction on the chorioallantoic membrane of the chick embryo. Archives of histology and cytology. 2010;73:45-53.

27. Zaugg P, Djonov V, Fuchtbauer EM, Draeger A. Sorting of murine vascular smooth muscle cells during wound healing in the chicken chorioallantoic membrane. Experimental cell research. 1999;253:599-606.

28. Ribatti D, Vacca A, Ranieri G, Sorino S, Roncali L. The Chick Embryo Chorioallantoic Membrane as an in vivo Wound Healing Model. Pathology, research and practice. 1996;192:1068-76.

29. Bertossi M, Girolamo F, Errede M, Virgintino D, Roncali L. Effects of 6-aminonicotinamide gliotoxin on blood-brain barrier differentiation in the chick embryo cerebellum. Anatomy and embryology. 2003;207:209-19.

30. Janzer RC. The blood-brain barrier: cellular basis. Journal of inherited metabolic disease. 1993;16:639-47.

31. Gabrielli MG, Accili D. The chick chorioallantoic membrane: a model of molecular, structural, and functional adaptation to transepithelial ion transport and barrier function during embryonic development. Journal of biomedicine \& biotechnology. 2010;2010:940741.

32. DeFouw DO, Rizzo VJ, Steinfeld R, Feinberg RN. Mapping of the microcirculation in the chick chorioallantoic membrane during normal angiogenesis. Microvascular research. 1989;38:136-47.

33. Deryugina EI, Quigley JP. Chick embryo chorioallantoic membrane model systems to study and visualize human tumor cell metastasis. Histochemistry and cell biology. 2008;130:1119-30.

34. Tufan AC, Satiroglu-Tufan NL. The chick embryo chorioallantoic membrane as a model system for the study of tumor angiogenesis, invasion and development of anti-angiogenic agents. Curr Cancer Drug Tar. 2005;5:249-66.

35. Richardson M, Singh G. Observations on the use of the avian chorioallantoic membrane (CAM) model in investigations into angiogenesis. Current Drug Targets-Cardiovascular \& Hematological Disorders. 2003;3:155-85.

36. Fuchs A, Lindenbaum ES. The two- and three-dimensional structure of the microcirculation of the chick chorioallantoic membrane. Acta anatomica. 1988;131:271-5.

37. Shumko JZ, Defouw DO, Feinberg RN. Vascular histodifferentiation in the chick chorioallantoic membrane: a morphometric study. The Anatomical record. 1988;220:179-89.

38. Ausprunk DH, Knighton DR, Folkman J. Differentiation of vascular endothelium in the chick chorioallantois: a structural and autoradiographic study. Developmental biology. 1974;38:237-48.

39. Korostyshevskaia IM, Maksimov VF. How chicken embryo survives after half of shell is sealed? Ontogenez. 2009;40:136-47.

40. Maksimov VF, Korostyshevskaia IM. Developmental peculiarities of gas transporting system in chick embryo under respiratory surface restriction. Rossiiskii fiziologicheskii zhurnal imeni IM Sechenova / Rossiiskaia akademiia nauk. 2007;93:1413-22

41. Wagner-Amos K, Seymour RS. Effect of regional changes to shell conductance on oxygen consumption and growth of chicken embryos. Respir Physiol. 2002;129:385-95.

42. Akins RE, Tuan RS. Transepithelial calcium transport in the chick chorioallantoic membrane. I. Isolation and characterization of chorionic ectoderm cells. Journal of cell science. 1993;105 ( Pt 2):369-79.

43. Akins RE, Tuan RS. Transepithelial calcium transport in the chick chorioallantoic membrane. II. Compartmentalization of calcium during uptake. Journal of cell science. 1993;105 ( Pt 2):381-8.

44. Tuan RS. Mechanism and regulation of calcium transport by the chick embryonic chorioallantoic membrane. The Journal of experimental zoology Supplement : published under auspices of the American Society of Zoologists and the Division of Comparative Physiology and Biochemistry / the Wistar Institute of Anatomy and Biology. 1987;1:1-13.

45. Tuan RS. Supplemented eggshell restores calcium transport in chorioallantoic membrane of cultured shell-less chick embryos. Journal of embryology and experimental morphology. 1983;74:119-31.

46. Tuan RS. Calcium transport and related functions in the chorioallantoic membrane of cultured shell-less chick embryos. Dev Biol. 1980;74:196-204.

47. Gabrielli MG, Cox JV, Materazzi G, Menghi G. Cell type-specific and developmentally regulated expression of the AE1 anion exchanger in the chicken chorioallantoic membrane. Histochemistry and cell biology. 2004;121:189-99.

48. Gabrielli MG. Carbonic anhydrases in chick extra-embryonic structures: A role for CA in bicarbonate reabsorption through the chorioallantoic membrane. Journal of Enzyme Inhibition and Medicinal Chemistry. 2004;19:283-86.

49. Saleuddin AS, Kyriakides CP, Peacock A, Simkiss K. Physiological and ultrastructural aspects of ion movements across the chorioallantois. Comparative biochemistry and physiology A, Comparative physiology. 1976;54:7-12.

50. Stewart ME, Terepka AR. Transport functions of the chick chorio-allantoic membrane. I. Normal histology and evidence for active electrolyte transport from the allantoic fluid, in vivo. Experimental cell research. 1969;58:93-106.
51. Balciuniene N, Tamasauskas A, Valanciute A, et al. Histology of human glioblastoma transplanted on chicken chorioallantoic membrane. Medicina. 2009;45:123-31.

52. Hurst EW, Barbara C, McLennan GC. A note on the survival and growth of human and rabbit tissues (normal and neoplastic) on the chorio-allantois of the chick and duck embyo. Australian Journal of Experimental Biology and Medical Science. 1939;:17.

53. Vogel HB, Berry RG. Chorioallantoic membrane heterotransplantation of human brain tumors. International journal of cancer Journal international du cancer. 1975; 15:401-8.

54. Tereseviciute N, Tamasauskas A, Valanciute A, Deltuva V, von graf KD. Evaluation of morphological issues of central nervous system glioblastoma on chicken embryo chorioallantoic membrane. Polish journal of veterinary sciences. 2007;10:173-8.

55. Strojnik T, Kavalar R, Barone TA, Plunkett RJ. Experimental model and immunohistochemical comparison of U87 human glioblastoma cell xenografts on the chicken chorioallantoic membrane and in rat brains. Anticancer research. 2010;30:4851-60.

56. He YQ, Li Y, Wang XY, et al. Dimethyl phenyl piperazine iodide (DMPP) induces glioma regression by inhibiting angiogenesis. Exp Cell Res. 2014;320:354-64.

57. Martinho O, Silva-Oliveira R, Miranda-Goncalves V, et al. In Vitro and In Vivo Analysis of RTK Inhibitor Efficacy and Identification of Its Novel Targets in Glioblastomas. Translational oncology. 2013;6:187-96.

58. Nico B, Annese T, Guidolin D, Finato N, Crivellato E, Ribatti D. Epo is involved in angiogenesis in human glioma. Journal of neuro-oncology. 2011;102:51-8

59. Hagedorn M, Javerzat S, Gilges D, et al. Accessing key steps of human tumor progression in vivo by using an avian embryo model. P Natl Acad Sci USA. 2005;102:1643-48.

60. Pen A, Durocher Y, Slinn J, et al. Insulin-like growth factor binding protein 7 exhibits tumor suppressive and vessel stabilization properties in U87MG and T98G glioblastoma cell lines. Cancer biology \& therapy. 2011;12:634-46.

61. Ribatti D. Erythropoietin, the first century. Leukemia research. 2008;32:1169-72.

62. Said HM, Hagemann C, Staab A, et al. Expression patterns of the hypoxia-related genes osteopontin, CA9, erythropoietin, VEGF and HIF-1alpha in human glioma in vitro and in vivo. Radiotherapy and oncology : journal of the European Society for Therapeutic Radiology and Oncology. 2007;83:398-405.

63. Jiang L, Song L, Wu J, et al. Bmi-1 promotes glioma angiogenesis by activating NF-kappaB signaling. PloS one. 2013;8:e55527.

64. Li J, Gong LY, Song LB, et al. Oncoprotein Bmi-1 renders apoptotic resistance to glioma cells through activation of the IKK-nuclear factor-kappaB Pathway. The American journal of pathology. 2010;176:699-709.

65. Auf G, Jabouille A, Guerit S, et al. Inositol-requiring enzyme 1alpha is a key regulator of angiogenesis and invasion in malignant glioma. Proceedings of the National Academy of Sciences of the United States of America. 2010;107:15553-8.

66. Teruszkin Balassiano I, Alves de Paulo S, Henriques Silva N, et al. Effects of perillyl alcohol in glial C6 cell line in vitro and anti-metastatic activity in chorioallantoic membrane model. International journal of molecular medicine. 2002;10:785-8.

67. Durupt F, Koppers-Lalic D, Balme B, et al. The chicken chorioallantoic membrane tumor assay as model for qualitative testing of oncolytic adenoviruses. Cancer gene therapy. 2012;19:58-68

68. Grodzik M, Sawosz E, Wierzbicki M, et al. Nanoparticles of carbon allotropes inhibit glioblastoma multiforme angiogenesis in ovo. International journal of nanomedicine. 2011;6:3041-8.

69. Shoin K, Yamashita J, Enkaku F, Sasaki T, Tanaka M, Endo Y. Chick embryo assay as chemosensitivity test for malignant glioma. Japanese journal of cancer research : Gann. 1991;82:1165-70.

70. Gualandris A, Rusnati M, Belleri M, et al. Basic fibroblast growth factor overexpression in endothelial cells: an autocrine mechanism for angiogenesis and angioproliferative diseases. Cell growth \& differentiation : the molecular biology journal of the American Association for Cancer Research. 1996;7:147-60.

71. Kronenburg A, Braun KP, van der Zwan A, Klijn CJ. Recent advances in moyamoya disease: pathophysiology and treatment. Current neurology and neuroscience reports. 2014;14:423.

72. Janzer RC, Raff MC. Astrocytes induce blood-brain barrier properties in endothelial cells. Nature. 1987;325:253-7.

73. Schlosshauer B, Herzog KH. Neurothelin: an inducible cell surface glycoprotein of blood-brain barrier-specific endothelial cells and distinct neurons. The Journal of cell biology. 1990;110:1261-74.

74. Lobrinus JA, Juillerat-Jeanneret L, Darekar P, Schlosshauer B, Janzer RC. Induction of the blood-brain barrier specific HT7 and neurothelin epitopes in endothelial cells of the chick chorioallantoic vessels by a soluble factor derived from astrocytes. Brain research Developmental brain research. 1992;70:207-11.

75. Holash JA, Stewart PA. Chorioallantoic membrane (CAM) vessels do not respond to blood-brain barrier (BBB) induction. Advances in experimental medicine and biology. 1993;331:223-8.

76. Holash JA, Noden DM, Stewart PA. Re-evaluating the role of astrocytes in blood-brain barrier induction. Developmental dynamics : an official publication of the American Association of Anatomists. 1993:197:14-25. 